\title{
Favorable outcomes of localized synovial sarcoma patients with a high utilization rate of neoadjuvant and/or adjuvant chemotherapy
}

\author{
HIDETATSU OUTANI ${ }^{1}$, SHIGEKI KAKUNAGA ${ }^{2}$, KENICHIRO HAMADA ${ }^{1}$, SATOSHI TAKENAKA ${ }^{1}$, \\ YOSHINORI IMURA $^{3}$, SHIGENORI NAGATA ${ }^{4}$, TAKAAKI TANAKA ${ }^{3}$, HIRONARI TAMIYA ${ }^{3}$, KAZUYA OSHIMA $^{5}$, \\ NORIFUMI NAKA ${ }^{3}$, IKUO KUDAWARA ${ }^{2}$, NOBUHITO ARAKI ${ }^{6}$, TAKAFUMI UEDA ${ }^{2}$ and HIDEKI YOSHIKAWA ${ }^{1}$ \\ ${ }^{1}$ Department of Orthopaedic Surgery, Osaka University Graduate School of Medicine, Suita, Osaka 565-0871; \\ ${ }^{2}$ Department of Orthopaedic Surgery, Osaka National Hospital, Chuo-ku, Osaka 540-0006; \\ ${ }^{3}$ Musculoskeletal Oncology Service, Osaka International Cancer Institute, Chuo-ku, Osaka 541-8567; \\ ${ }^{4}$ Department of Diagnostic Pathology and Cytology, Osaka International Cancer Institute, Chuo-ku, Osaka 541-8567; \\ ${ }^{5}$ Department of Orthopaedic Surgery, Bell-land General Hospital, Sakai, Osaka 599-8247; \\ ${ }^{6}$ Department of Orthopaedic Surgery, Ashiya Municipal Hospital, Ashiya, Hyogo 659-8502, Japan
}

Received December 18, 2018; Accepted April 17, 2019

DOI: $10.3892 / \mathrm{mco} .2019 .1863$

\begin{abstract}
Synovial sarcoma (SS) is considered to be a chemosensitive, soft tissue sarcoma. Therefore, neoadjuvant and/or adjuvant chemotherapy (N/AC) is used for the treatment of high-risk SS patients. However, the role of N/AC remains controversial. The present study aimed to review the clinical outcomes of surgically treated localized SS and investigate the effects of N/AC with long-term observation. The clinical outcomes of 54 patients with surgically treated localized SS were retrospectively analyzed. The median patient age was 42 years (range, 8-81 years), and the median follow-up period was 94 months for survivors (range, 7-220 months). A total of 38 patients (70\%) received chemotherapy. Of these, 32 (59\%) patients received neoadjuvant chemotherapy, $33(61 \%)$ received adjuvant chemotherapy, and 27 (50\%) received neoadjuvant and adjuvant chemotherapy. Fourteen patients (26\%) received adjuvant radiotherapy. Three patients $(6 \%)$ had local recurrence and 13 patients (24\%) developed distant metastasis. The overall survival (OS) rates at 5 and 10 years were 87 and $84 \%$, respectively. N/AC did not improve survival. In conclusion, we found satisfactory long-term OS among patients with a high utilization rate of N/AC. Further study should be necessary to evaluate which population of SS would benefit from N/AC.
\end{abstract}

Correspondence to: Dr Hidetatsu Outani, Department of Orthopaedic Surgery, Osaka University Graduate School of Medicine, 2-2 Yamadaoka, Suita, Osaka 565-0871, Japan E-mail: h-otani@ort.med.osaka-u.ac.jp

Key words: adjuvant chemotherapy, long-term outcomes, neoadjuvant chemotherapy, synovial sarcoma

\section{Introduction}

Synovial sarcoma (SS) is a soft tissue sarcoma (STS) that can occur at many different locations. In Japan, SS has been ranked as the fifth most common STS, with the exception of well-differentiated liposarcoma, and its occurrence has been shown to peak in the third and fourth decades of life (1). The standard treatment for SS is wide surgical resection with or without radiotherapy, which is similar to the approach for other STSs (2). The role of chemotherapy is highly debated. A review of 15 clinical trials demonstrated that advanced SS had a better response to systemic chemotherapy than other subtypes of STS (3). Hence, neoadjuvant and/or adjuvant chemotherapy (N/AC) is frequently used for localized SS, especially in young patients (4). However, the role of N/AC is still controversial. Several large studies have demonstrated a survival benefit with N/AC (5-7), whereas others have not demonstrated such a benefit (4,8-10). Further, the dose efficacy of N/AC remains unclear. The present study aimed to review the clinical outcomes of surgically treated localized SS and investigate the effects of N/AC with long-term observation.

\section{Patients and methods}

We assessed our institutional database and identified 54 patients with histological diagnosis of localized SS treated between 2000 and 2016. All patients were staged according to computed tomography (CT) and/or magnetic resonance imaging (MRI) findings. The 8th American Joint Committee on Cancer (AJCC) staging system was used for disease staging (11). The greatest dimension of the tumor was defined as tumor size. Tumor grading was evaluated using the French Federation of Cancer Centres Sarcoma Group (FNCLCC) grading system (12). According to the FNCLCC grading system, the tumor could be either grade 2 or grade 3 , 
depending on the mitotic rate, the extent of necrosis, or both; however, no grading information was available. The resection margin status was classified as R0 (macroscopically and microscopically clear), R1 (macroscopically clear and microscopically involved), and R2 (macroscopically involved). Inclusion criteria of N/AC were deep located and $\geq 5 \mathrm{~cm}$ tumors. If tumor was attached to critical structures, or seemed to contaminate surrounding tissue after unplanned surgery, the use of N/AC depended on the physician's choice. Exclusion criteria of N/AC was superficial located and $<5 \mathrm{~cm}$ tumors and patients $>70$ years old. The administered regimen was doxorubicin $\left(60 \mathrm{mg} / \mathrm{m}^{2}\right)$ in combination with ifosfamide $\left(6-10 \mathrm{~g} / \mathrm{m}^{2}\right)$, high-dose ifosfamide $\left(12-15 \mathrm{~g} / \mathrm{m}^{2}\right)$, or both. When histology showed the existence of poorly differentiated component, regimens administered were doxorubicin $\left(60 \mathrm{mg} / \mathrm{m}^{2}\right)$ in combination with vincristine $\left(1.5 \mathrm{mg} / \mathrm{m}^{2}\right)$ and cyclophosphamide $\left(1,000 \mathrm{mg} / \mathrm{m}^{2}\right)$, ifosfamide $\left(5 \mathrm{~g} / \mathrm{m}^{2}\right)$ in combination with etoposide $\left(300 \mathrm{mg} / \mathrm{m}^{2}\right)$. If surgical margin was contaminated, or close to the tumor, radiotherapy was used based on physician's choice. Patients were followed up at our outpatient clinic every 3 months during first 2 years, every 6 months during next 3 years, then annually until 10 years had passed or death. At each visit, chest radiographs were obtained. Chest CT and/or local MRI were performed at intervals of 3-6 months until 5 years. This study was approved by Osaka University Clinical Research Review Committee, and waived off the requirement for written informed consent from the subjects (certificate no. 14240-2).

Statistical analysis. We assessed local recurrence free survival (LRFS), overall survival (OS), and metastasis-free survival (MFS) using the Kaplan-Meier method with 95\% confidence intervals (CIs). Differences between LRFS, OS, and MFS were compared using the log-rank test. Descriptive statistics were used to show the distribution of variables in the population. All statistical analyses were performed using SPSS 23.0 software (IBM Corp., Armonk, NY, USA). P $<0.05$ was considered to indicate a statistically significant difference.

\section{Results}

Patient and treatment demographics. The study included 54 patients ( 23 male and 31 female patients). The median patient age was 42 years (range, 8-81 years), and the median follow-up period was 94 months for survivors (range, 7-220 months). Fifteen patients were referred after unplanned surgery, 36 had initial definitive treatment at our institutions, and 3 had no records about initial treatment before reference. All patients underwent surgical resection of the primary tumor. Six patients (11\%) had positive surgical margins. Of the 6 patients, 3 had R2 resection and 3 had R1 resection. Fourteen patients (26\%) received radiotherapy after surgery. Thirty-eight patients (70\%) were treated with chemotherapy. Neoadjuvant chemotherapy and adjuvant chemotherapy were used in 32 patients (59\%) and 33 patients (61\%), respectively. Twenty-seven (50\%) patients received both neoadjuvant and adjuvant chemotherapy. All patients with stage III tumors, except 3 patients, received N/AC. One patient did not receive chemotherapy because of high age ( 81 years) and the other patients because of superficial location. Their tumor size were
Table I. Clinical and treatment characteristics.

\begin{tabular}{|c|c|c|c|}
\hline \multirow[b]{2}{*}{ Variables } & \multicolumn{2}{|c|}{ All patients } & \multirow[b]{2}{*}{ P-value } \\
\hline & N/AC (-) & N/AC (+) & \\
\hline Sex & & & 0.911 \\
\hline Male & 7 & 16 & \\
\hline Female & 9 & 22 & \\
\hline Age & & & 0.233 \\
\hline$<40$ & 6 & 21 & \\
\hline $40 \leq$ & 10 & 17 & \\
\hline Unplanned surgery & & & 0.559 \\
\hline Yes & 6 & 9 & \\
\hline No & 9 & 27 & \\
\hline ND & 1 & 2 & \\
\hline Grade & & & 0.005 \\
\hline 2 & 11 & 15 & \\
\hline 3 & 1 & 20 & \\
\hline ND & 4 & 3 & \\
\hline Depth & & & 0.008 \\
\hline Superficial & 6 & 3 & \\
\hline Deep & 10 & 35 & \\
\hline Size & & & $<0.001$ \\
\hline$<5 \mathrm{~cm}$ & 12 & 9 & \\
\hline $5 \mathrm{~cm} \leq$ & 3 & 29 & \\
\hline Site & & & 0.981 \\
\hline Limbs & 11 & 26 & \\
\hline Axial & 5 & 12 & \\
\hline Stage & & & $<0.001$ \\
\hline II & 13 & 9 & \\
\hline III & 3 & 29 & \\
\hline Surgical margin & & & 0.246 \\
\hline Negative & 13 & 35 & \\
\hline Positive & 3 & 3 & \\
\hline RT & & & 0.562 \\
\hline Yes & 5 & 9 & \\
\hline No & 11 & 29 & \\
\hline
\end{tabular}

N/AC, neoadjuvant and/or adjuvant chemotherapy; ND, no data; RT, radiotherapy. $\chi^{2}$ test was used to analyze the relationships between the variables.

8 and $10 \mathrm{~cm}$. The factors associated with receipt of N/AC were grade 3 histology, deep-seated location, tumor size $\geq 5 \mathrm{~cm}$, and stage III tumor (Table I). The factors associated with receipt of radiotherapy were positive surgical margins, age $\geq 40$, and grade 3 tumor $(\mathrm{P}=0.001, \mathrm{P}=0.062$, and $\mathrm{P}=0.08$, respectively; data not shown).

Outcomes. Three patients (6\%) had local recurrence (LR) and 13 patients $(24 \%)$ developed distant metastasis. Of the 13 patients who developed distant metastasis, 8 died of SS, 4 showed no evidence of disease after metastasectomy, and one lost follow-up due to shifting to palliative care. 

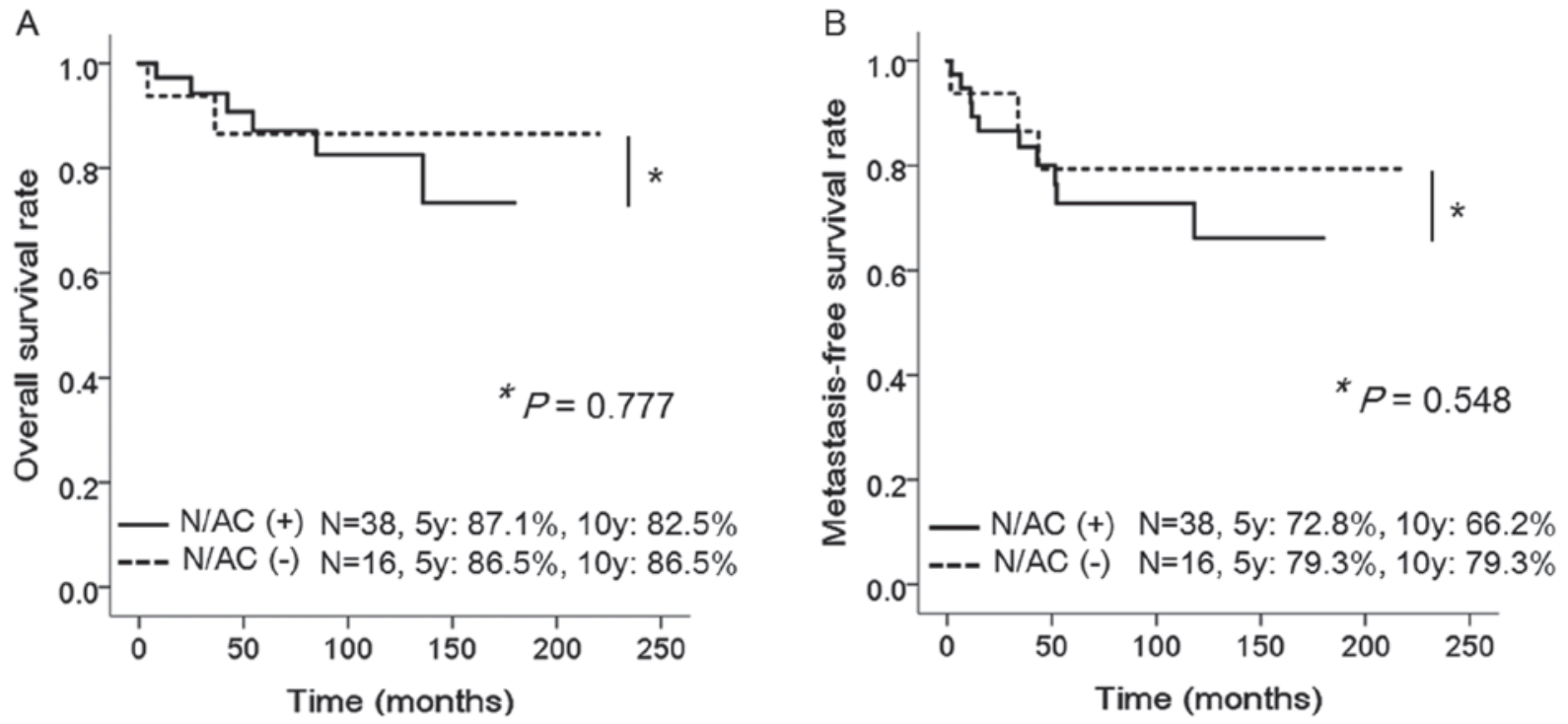

Figure 1. (A) Overall survival in all patients who received (continuous line) and those who did not receive (broken line) neoadjuvant and/or adjuvant chemotherapy. No significant difference is observed between the groups $(\mathrm{P}=0.777)$. (B) Metastasis-free survival in all patients who received (continuous line) and those who did not receive (broken line) neoadjuvant and/or adjuvant chemotherapy. No significant difference is observed between the groups $(\mathrm{P}=0.548)$.

One patient died of another cause. The 5- and 10-year LR rates were $93.7 \%$ (95\% CI, 86.8-100) and 93.7\% (95\% CI, $86.8-100)$, respectively. Tumor size $<5 \mathrm{~cm}$ was associated with worse LRFS $(\mathrm{P}=0.033)$. The 5- and 10-year MFS rates were $74.7 \%$ (95\% CI, 62.2-87.2) and 70.0\% (95\% CI, 55.3-84.7), respectively. Tumor size $\geq 5 \mathrm{~cm}$, and receipt of radiotherapy were significantly associated with worse MFS $(\mathrm{P}=0.045$, and $\mathrm{P}=0.02$, respectively). The 5- and 10-year OS rates were 87.1\% (95\% CI, 77.6-96.9) and 83.7\% (95\% CI, 72.3-95.1), respectively. Receipt of radiotherapy was significantly associated with worse OS $(\mathrm{P}=0.02)$ (Table II), probably due to selection bias. Because receipt of radiotherapy was associated with positive surgical margins, age $\geq 40$, and grade 3 tumor $(\mathrm{P}=0.001, \mathrm{P}=0.062$, and $\mathrm{P}=0.08$, respectively; data not shown) N/AC did not have significant effects on LR, MFS, and $\mathrm{OS}(\mathrm{P}=0.131, \mathrm{P}=0.548$, and $\mathrm{P}=0.777$, respectively; Fig. 1$)$.

\section{Discussion}

In the present study, the 5- and 10-year OS rates were found to be 87.1 and $83.7 \%$, respectively. To our knowledge, this is the best long-term result among published large series involving adult SS patients (Table III), although Krieg et al (13) reported that SS tends to develop metastasis late with high mortality. There are 3 possible factors to explain the favorable outcomes. The first is patient and tumor demographics. A number of prognostic factors for localized SS have been reported, and among them, the relatively common favorable factors were small tumor size and young age at diagnosis $(4,5,7-9,14)$. It remains unclear whether the prognostic significance of age is related to biological variables or to historically different treatment approaches adapted in pediatric vs. adult patients (15). The proportion of tumors measuring $\geq 5 \mathrm{~cm}$ and the median age at diagnosis in this cohort were comparable to the values in previous studies (Table III). Thus, patient and tumor demographics appear to have less effect on the outcomes. The second is primary treatment. In this study, the status of the surgical margin was comparable to that in previous studies and the rate of adjuvant radiotherapy use was lower than that in previous studies (Table III). On the contrary, the proportion of patients who received N/AC was higher than that in previous studies (Table III). The third is treatment after tumor relapse. For the last 3-4 decades, there have been few options other than doxorubicin and ifosfamide for the treatment of advanced STS. Recently, 3 new drugs (pazopanib, trabectedin, and eribulin) were approved in Japan for the treatment of advanced STS. The effects of these new drugs on SS have not been clarified yet, but it is possible that these new drugs will prolong the survival of patients with relapsed SS. Furthermore, 4 patients achieved disease free survival after metastasectomy. Hence aggressive treatment after tumor relapse including local and systematic therapy likely contributed to prolong the survival. However, considering that the rates of local recurrence and distant metastasis were lower in this study than those in previous studies (Table III), primary treatment appears to have a more vital role than treatment after tumor relapse. Taken together, the effect of N/AC on survival is considered important in this cohort.

The effect of N/AC on SS has not been proven previously, and study results have been conflicting. Italiano et al (10) demonstrated that N/AC did not have a significant impact on OS, using the French Sarcoma Group Database. On the contrary, Chen et al (16) reported that adjuvant chemotherapy improved disease-specific survival and prolonged the time to metastasis in stage IIB/III SS patients. Recently, Vining et al (17) reported improved outcomes with adjuvant chemotherapy in stage III SS patients from an analysis of the National Cancer Database and recommended less restricted use of adjuvant chemotherapy for stage III SS. These differences might come from varying use of chemotherapy regimens among patients and different proportions of N/AC administration. Besides which population of stage III SS could improve survival by N/AC is still unclear. In this study, all patients with stage III SS, except 3 patients, received N/AC. Of the 3 patients, one died of disease 
Table II. Univariate analysis of factors influencing LRFS, MFS, and OS.

Variables LRFS P-value MFS P-value OS P-value

\section{Sex}

Male

0.703

0.511

0.239

Female

Age

$<40$

0.608

0.598

0.357

$40 \leq$

Unplanned surgery

Yes

0.331

0.061

0.234

No

ND

Site

Limbs

0.198

0.071

0.343

Axial

Size

$<5 \mathrm{~cm}$

0.033

0.045

0.093

$5 \mathrm{~cm} \leq$

Depth

Superficial

0.42

0.084

0.193

Deep

Histology

\begin{tabular}{llll} 
Monophasic & 0.635 & 0.272 & 0.915 \\
Biphasic & & & \\
NOS & & & \\
Grade & & 0.191 & 0.340 \\
2 & 0.513 & & \\
3 & & & \\
ND & & 0.068 & 0.118 \\
Surgical margin & 0.12 & & \\
Negative & & & \\
Positive & & 0.020 \\
RT & & & \\
Yes & 0.601 & & 0.777 \\
No & & 0.548 & \\
N/AC & & & \\
Yes & 0.131 & & \\
No & & & \\
\hline
\end{tabular}

LRFS, local recurrence free survival; MFS, metastasis-free survival; OS, overall survival; RT, radiotherapy; N/AC, neoadjuvant and/or adjuvant chemotherapy; ND, no data. Differences between LRFS, MFS, and OS were compared using the log-rank test.

4 months after surgery, 2 were continuous disease free for 69 and 50 months after surgery. Thus, we could not compare the effects of N/AC in high-risk patients properly and recommend that all stage III patients should have N/AC. Nonetheless, the treatment resulted in better long-term outcomes than those of previous studies. Collectively, our results suggest that patients with stage III SS include the population of patients who benefit

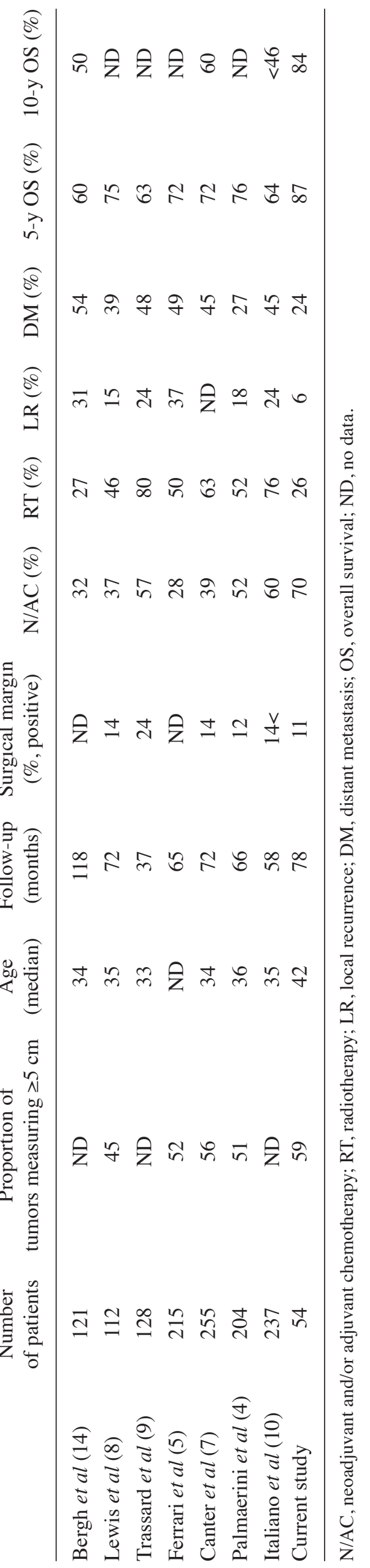


from N/AC and high utilization of N/AC in SS might improve long-term outcomes.

Some studies have shown that adjuvant radiotherapy improved survival in SS patients $(18,19)$. However, we showed that radiotherapy had adverse effects on OS and MFS. Receipt of radiotherapy was associated with positive surgical margins, age $\geq 40$, and grade 3 tumor $(\mathrm{P}=0.001, \mathrm{P}=0.062$, and $\mathrm{P}=0.08$, respectively; data not shown); thus, the adverse effects of radiotherapy observed in this study resulted from selection bias and we were not able to evaluate the impact of radiotherapy appropriately. In this study, radiotherapy and surgical margin did not correlate with LRFS probably due to low rate of LR. Surprisingly, tumor size $<5 \mathrm{~cm}$ was associated with LRFS. Because 2 of the 3 patients who developed LR had unplanned surgery, we speculated the correlation might be affected by unplanned surgery.

The present study has several limitations. The number of patients was too small to draw a definitive conclusion. There were no information of chromosomal translocation. We did not use fluorescent in situ hybridization for diagnosis, and that might affect the patient population. We were not able to obtain histological grading information in some cases. It was sometimes difficult to determine FNCLCC grading after neoadjuvant chemotherapy. Administration regimens were not uniform. The rate of positive surgical margins was comparable to that reported in previous series, but the rate was the lowest among previously reported rates. This might be associated with lower local recurrence rates and might influence favorable outcomes. The rate of radiotherapy use was low. Usually, radiotherapy is considered useful for local control, and it can improve survival in SS patients (19). We considered that the low rate of radiotherapy use did not lead to overestimation of the effects of N/AC. Finally, there was possible selection bias with regard to receipt of N/AC. We delivered N/AC to almost all high-risk patients, and we failed to assess the effects of N/AC appropriately.

In conclusion, we demonstrated satisfactory long-term outcomes in localized SS patients with a high utilization rate of N/AC. We failed to show the impact of N/AC on survival probably due to high use of N/AC on stage III patients. Further study should be necessary to evaluate which population of SS would benefit from N/AC.

\section{Acknowledgements}

Not applicable.

\section{Funding}

No funding was received.

\section{Availability of data and materials}

The datasets used in the present study are available from the corresponding author on reasonable request.

\section{Authors' contributions}

HO conceived and designed the study, collected, analyzed, and interpreted the data, and wrote the manuscript. SK analyzed data and revised the manuscript. $\mathrm{KH}$ and ST collected data and reviewed the manuscript. SN reviewed the histological grading of the tumor specimens. YI, TT, HT, and KO collected and interpreted data. NN, IK, NA, TU, and HY analyzed and interpreted the data and reviewed and revised the manuscript. All authors read and approved the final manuscript.

\section{Ethics approval and consent to participate}

The present study was approved by Osaka University Clinical Research Review Committee, and waived off the requirement for written informed consent from the subjects (certificate no. 14240-2).

\section{Patient consent for publication}

Not applicable.

\section{Competing interests}

The authors declare that they have no conflict of interest.

\section{References}

1. Ogura K, Higashi T and Kawai A: Statistics of soft-tissue sarcoma in Japan: Report from the bone and soft tissue tumor registry in Japan. J Orthop Sci 22: 755-764, 2017.

2. Pisters PW, O'Sullivan B and Maki RG: Evidence-based recommendations for local therapy for soft tissue sarcomas. J Clin Oncol 25: 1003-1008, 2007.

3. Vlenterie M, Litière S, Rizzo E, Marréaud S, Judson I, Gelderblom H, Le Cesne A, Wardelmann E, Messiou C, Gronchi A and van der Graaf WT: Outcome of chemotherapy in advanced synovial sarcoma patients: Review of 15 clinical trials from the European organisation for research and treatment of cancer soft tissue and bone sarcoma group; setting a new landmark for studies in this entity. Eur J Cancer 58: 62-72, 2016.

4. Palmerini E, Staals EL, Alberghini M, Zanella L, Ferrari C, Benassi MS, Picci P, Mercuri M, Bacci G and Ferrari S: Synovial sarcoma: Retrospective analysis of 250 patients treated at a single institution. Cancer 115: 2988-2998, 2009.

5. Ferrari A, Gronchi A, Casanova M, Meazza C, Gandola L, Collini P, Lozza L, Bertulli R, Olmi P and Casali PG: Synovial sarcoma: A retrospective analysis of 271 patients of all ages treated at a single institution. Cancer 101: 627-634, 2004.

6. Eilber FC, Brennan MF, Eilber FR, Eckardt JJ, Grobmyer SR, Riedel E, Forscher C, Maki RG and Singer S: Chemotherapy is associated with improved survival in adult patients with primary extremity synovial sarcoma. Ann Surg 246: 105-113, 2007.

7. Canter RJ, Qin LX, Maki RG, Brennan MF, Ladanyi M and Singer S: A synovial sarcoma-specific preoperative nomogram supports a survival benefit to ifosfamide-based chemotherapy and improves risk stratification for patients. Clin Cancer Res 14: 8191-8197, 2008.

8. Lewis JJ, Antonescu CR, Leung DH, Blumberg D, Healey JH, Woodruff JM and Brennan MF: Synovial sarcoma: A multivariate analysis of prognostic factors in 112 patients with primary localized tumors of the extremity. J Clin Oncol 18: 2087-2094, 2000.

9. Trassard M, Le Doussal V, Hacène $K$, Terrier $P$, Ranchère D, Guillou L, Fiche M, Collin F, Vilain MO, Bertrand G, et al: Prognostic factors in localized primary synovial sarcoma: A multicenter study of 128 adult patients. J Clin Oncol 19: 525-534, 2001.

10. Italiano A, Penel N, Robin YM, Bui B, Le Cesne A, Piperno-Neumann S, Tubiana-Hulin M, Bompas E, Chevreau C, Isambert $\mathrm{N}$, et al: Neo/adjuvant chemotherapy does not improve outcome in resected primary synovial sarcoma: A study of the French sarcoma group. Ann Oncol 20: 425-430, 2009.

11. Amin M, Edge S, Greene F, Byrd DR, Brookland RK, Washington MK, Gershenwald JE, Compton CC, Hess KR Sullivan DC, et al (eds): AJCC Cancer Staging Manual (8th edition). New York, Springer, 2017. 
12. Trojani M, Contesso G, Coindre JM, Rouesse J, Bui NB, de Mascarel A, Goussot JF, David M, Bonichon F and Lagarde C: Soft-tissue sarcomas of adults; study of pathological prognostic variables and definition of a histopathological grading system. Int J Cancer 33: 37-42, 1984.

13. Krieg AH, Hefti F, Speth BM, Jundt G, Guillou L, Exner UG, von Hochstetter AR, Cserhati MD, Fuchs B, Mouhsine E, et al: Synovial sarcomas usually metastasize after $>5$ years: A multicenter retrospective analysis with minimum follow-up of 10 years for survivors. Ann Oncol 22: 458-467, 2011

14. Bergh P, Meis-Kindblom JM, Gherlinzoni F, Berlin O, Bacchini P, Bertoni F, Gunterberg B and Kindblom LG: Synovial sarcoma: Identification of low and high risk groups. Cancer 85: 2596-2607,1999.

15. Vlenterie M, Ho VK, Kaal SE, Vlenterie R, Haas R and van der Graaf WT: Age as an independent prognostic factor for survival of localised synovial sarcoma patients. Br J Cancer 113: 1602-1606, 2015.
16. Chen Y, Yang Y, Wang C and Shi Y: Adjuvant chemotherapy decreases and postpones distant metastasis in extremity stage IIB/III synovial sarcoma patients. J Surg Oncol 106: 162-168, 2012.

17. Vining CC, Sinnamon AJ, Ecker BL, Kelz RR, Fraker DL, Roses RE and Karakousis GC: Adjuvant chemotherapy in resectable synovial sarcoma. J Surg Oncol 116: 550-558, 2017.

18. Song S, Park J, Kim HJ, Kim IH, Han I, Kim HS and Kim S: Effects of adjuvant radiotherapy in patients with synovial sarcoma. Am J Clin Oncol 40: 306-311, 2017.

19. Naing KW, Monjazeb AM, Li CS, Lee LY, Yang A, Borys D and Canter RJ: Perioperative radiotherapy is associated with improved survival among patients with synovial sarcoma: A SEER analysis. J Surg Oncol 111: 158-164, 2015. 